\title{
Geometric and radiometric estimation in a structured-light 3D scanner
}

\author{
Daljit Singh Dhillon • Venu Madhav Govindu
}

Received: 31 May 2014 / Revised: 8 January 2015 / Accepted: 19 January 2015 / Published online: 27 February 2015

(C) Springer-Verlag Berlin Heidelberg 2015

\begin{abstract}
The key problems in a structured-light 3D scanner using a projector and camera are its geometric and radiometric calibration. A projector can be effectively represented using the familiar pin-hole model for cameras. However, since we cannot directly observe the projector plane, it cannot be calibrated like a camera. The first contribution of our paper is to develop a geometric calibration and 3D estimation method that utilises the projective geometric relationships available in a projector-camera pair, i.e., homography induced by a plane and invariance of cross-ratios. The lowdimensional parametric form of the homography averages out individual errors, resulting in a geometric calibration approach that is both simple to use and highly accurate. We present an extensive set of results to demonstrate the effectiveness of our approach and also characterise its accuracy. Second, we present a method for correcting systematic errors introduced due to the radiometric non-linearities present in commercial projectors. These errors are pronounced when only a few phase shifts are used in the sinusoidal coding scheme for structured-light scanners. We develop a cubic spline-based method to model and remove the effects due to these non-linearities. The efficacy of our model is demonstrated on real datasets.
\end{abstract}

A preliminary version of this work appeared as [1]. This work was carried out at the Indian Institute of Science.

\section{S. Dhillon $(\bowtie)$}

Institute of Computer Science and Applied Mathematics,

University of Bern, 3012 Bern, Switzerland

e-mail:djdhillon@gmail.com

\section{M. Govindu}

Department of Electrical Engineering, Indian Institute of Science,

Bengaluru 560012, India

e-mail: venu@iisc.ernet.in
Keywords 3D reconstruction - Structured-light scanner · Projector calibration · Homography · Cross-ratio . Radiometric distortion

\section{Introduction}

The accurate 3D measurement of objects has a variety of applications in areas such as industrial manufacture, prosthetics, archaeology and digital preservation of cultural artefacts. An accurate representation of surface geometry can be acquired by illuminating the object of interest by a sequence of projected intensity patterns and imaging with a camera. Unlike imaging using ambient light, such coded illumination in structured-light scanning results in a dense correspondence between the projector and camera planes. This in turn leads to a dense reconstruction of the imaged surface. With dense correspondences easily available in structured-light systems, the primary problems pertain to the geometric calibration of the projector-camera pair as well as radiometric calibration of the projector. While a projector-camera pair has the same projective geometry as between a pair of cameras, for its calibration we need to use indirect methods since in a projector the direction of light is reversed. Also, typically projectors suffer from radiometric and some geometric non-linearities that need to be corrected. The first contribution of this paper is a new method of projector-camera geometry calibration and 3D estimation using projective geometric relationships, i.e., homography induced by a plane and projective invariance of cross-ratios. Our approach develops a low-dimensional parametric representation for calibration thereby simplifying its estimation while improving accuracy. Our second contribution is a method that corrects for radiometric non-linearities present in the projector-camera system. These non-linearities manifest as systematic artefacts (distortions) in 3D recon- 
struction. In our approach, we model these artefacts using cubic splines thereby allowing us to correct for the distortions.

The existing approaches for geometric and radiometric calibration in the literature are presented in Sect. 2. We provide an overview of our reconstruction pipeline in Sect. 3 . In Sect. 4, we present a brief overview of the geometry we will utilise, i.e. two-view projective geometry and view-space codification in a projector-camera system. In Sect. 5, we develop our approach for geometric calibration and 3D estimation in a projector-camera pair. This is followed by an extensive set of qualitative and quantitative results demonstrating the efficacy of our method. In Sect. 7, we describe the effect of radiometric projector non-linearities on 3D shape recovered in a structured-light system when using sinusoidal phase codification. In Sect. 8, we develop our cubic splinebased modelling solution and present the results of using our method for correcting radiometric non-linearities.

\section{Related work}

In this section, we describe the relevant approaches to projector-camera calibration as well as radiometric correction in the literature. Our discussion is limited to calibration of structured-light projector-camera pairs and does not consider methods to calibrate cameras. While calibrating a projector-camera pair geometrically, it is a common practice to use reference plane/s to capture projective relations between camera and projector pixels $[2,4-13,16]$. Each reference plane is projected upon with illumination markers, stripes or coded patterns to establish reference maps between projector pixels and their respective images in the camera view. It is common to use sinusoidal patterns for such viewspace codification during calibration and then the respective maps are termed as Reference Phase Maps [5-7,16]. Reference maps may be used to either explicitly or implicitly calibrate the projector and the relative geometry between the camera-projector view pair.

Here, we first consider active scanning systems, where the projector-camera geometry is allowed to change during data acquisition. For such systems, the method of [2] proposes to build an uncalibrated reconstruction by imposing affine constraints that are an approximation to the underlying projective relationships using reference planes. In [3], an error function based on the relative deviation of two back-projected rays from a common triangulation plane is minimised, yielding a Euclidean reconstruction. Both these methods [2,3] involve computationally expensive non-linear minimization. In [4], only the camera parameters are allowed to change. This approach uses reference planes and solves for homographies using a planar grid pattern (of circular markers) translated precisely in a direction perpendicular to the mounted plane, thereby allowing for direct 3D reconstruction. While this approach avoids non-linear minimization, the projector pre-calibration and the camera self-calibration described are cumbersome steps and require a precisely controlled mechanical device for moving the calibration plane.

For most methods, including the one presented in this paper, the projector-camera geometry is fixed. The method of [5] explicitly estimates the calibration parameters of the projector. The authors project a sequence of sinusoidal fringe patterns on a calibration plane, while placing it in different positions. Using dense correspondences between the camera and the projector for a given position of the calibration plane, they construct its respective virtual image in projector's image plane. Finally, using the set of such virtual images, they calibrate the projector just like a camera. Similar to $[5,8]$ performs explicit projector calibration by generating virtual images of calibration markers in projector's viewspace. However, they use graycode patterns to establish dense correspondences for each calibration plane position. Furthermore, they compute local homographies for the neighbourhoods of each calibration marker in each plane position to improve respective estimated positions of calibration markers in the virtual images. Although [5,8] perform explicit calibration, these methods are cumbersome and computationally demanding as they need a large number of images for reliable estimation. In contrast to [5,8], other methods do not explicitly estimate the projector parameters $[6,7,16]$. Peng and Gupta [6] uses a set of reference planes and a fully calibrated camera to implicitly calibrate the projector. By minimising a least-squares error, the projector's optical centre is estimated and in the scanning step, the point location is estimated by defining the corresponding epipolar plane. While the authors of [6] have demonstrated their system to be highly accurate, their calibration process is laborious. Furthermore, their 3D estimation approach involves a computationally expensive 1D search along the epipolar line in the reference phase map for each reconstructed $3 \mathrm{D}$ point. In [16], the authors use a pair of reference planes to calibrate the projection planes corresponding to vertical light stripes projected from the projector. This approach uses an over-parametrisation of the underlying projector geometry and does not impose any consistency constraints. In [7], the authors proposed a cross-ratio based $3 \mathrm{D}$ reconstruction system that avoids estimation of the epipolar geometry. For each camera pixel, the authors determine the phase values for the unknown surface and for three reference parallel planes. Using the invariance of the cross-ratio, 3D depth is established.

Some of the recent approaches to calibration utilise the projective geometric relationship available between the camera and projector when viewing a planar object, i.e. the homography between camera and projector induced by a plane. For instance, [9] uses a calibration plane of known size with orthogonal edges. Observing this object allows for 
the identification of vanishing points which are used to calibrate the camera. Subsequently, as in our work in [1], a rectified pattern is projected onto the same calibration object and the new vanishing points are identified. These vanishing points are used to calibrate the projector. In [10], the authors invert the workflow of camera calibration to calibrate the projector. Once the homography from camera to projector is established, [10] iteratively adjusts a projected checkerboard pattern to coincide with detected corners in the camera image. Having established correspondences in the projector plane in this indirect fashion, [10] can carry out the calibration of the projector with standard camera calibration methods. In [11], the authors utilise the projection of colour patterns onto a printed chessboard to establish correspondences between the camera and projector planes. These correspondences are refined using multiple projection patterns until an accurate camera-projector calibration is achieved. Other related methods based on planar homography include $[12,13]$.

Apart from the geometric calibration step, we need to account for residual radiometric and geometric nonlinearities that always exist in projector-camera pairs. While our solution is detailed in Sect. 7, here we briefly describe the existing methods in the literature. In [6], the authors effectively model radiometric non-linearities implicitly with the use of a reference phase map. However, their method ignores the existing geometric non-linearities while estimating the projector centre. In [5], although the authors use an exhaustive mapping between the projector and the camera their method does not model radiometric non-linearities. This may result in wave-like artefacts due to systematic errors in phase estimation for sinusoidal codification. In contrast, [7] does handle geometric and radiometric non-linearities. However, their calibration method has a high image acquisition time and their 3D estimation involves a computationally demanding 1D search algorithm along with the need for interpolation. Another class of approaches explicitly model the error in phase estimation introduced by the non-linearity of the projector's radiometric response. In [14], the authors consider the case where the number of projected patterns used in phase decoding varies between 3 and 5. For these cases, they represent the phase error introduced by projector non-linearities as a sum of higher order harmonics. Subsequently, this phase error is cancelled out by iteratively estimating the amount of harmonic distortion and subtracting it from the observed phase map. Similarly, in [15], the authors analyse the effect of non-linear projector distortion (gamma distortion) as a series sum of harmonics which gives them an expression for the error introduced in phase measurement. Subsequently, the phase estimate can be corrected for this non-linear error term. Finally, we note that $[4,16]$ and many other methods completely ignore existing non-linearities.

In terms of final accuracy, there exists a trade-off between the complexity and precision of the calibration method and the ease and accuracy of the estimation of shape. While some methods $[3,6,7]$ are computationally expensive others have an elaborate calibration/scanning process $[5,6,16]$. Some require very precise control of the placement of calibration planes $[4,6,7]$. The methods of $[4,6,7,16]$ use an overparameterization of the relative projector-camera geometry, leading to inaccuracies in 3D estimation. Many methods ignore the radiometric non-linearities $[4,5,16]$ as they do not employ sinusoidal codification which reduces the acquisition cycle. We seek to address some of the issues with existing methods as outlined above. As explained in Sect. 5, we utilise projective geometric relationships to greatly simplify the calibration process. Our method is flexible and does not require that the calibration plane be moved precisely. The overall mapping between the camera and projector is handled in an implicit two-step process and the resulting 3D estimate is both simple and reliable. Additionally, we model and rectify the systematic errors in phase estimation due to radiometric non-linearities to enable sinusoidal phase codification for a faster acquisition cycle.

\section{Our reconstruction pipeline}

This section gives an overview of our reconstruction pipeline. Our camera-projector view pair is operated in two phases, namely: (a) calibration phase, and (b) 3D scanning phase.

Calibration phase: Calibration phase involves:

Camera calibration For calibrating the camera, we use the implementation provided by [19] of the well-known approach of [20].

Projector calibration We implicitly calibrate the projector using a pair of reference planes to estimate Reference Maps. This is explained in detail in Sect. 5.

System calibration We also calibrate the projectorcamera relative geometry implicitly using the same Reference Maps as above.

Radiometric calibration We calibrate the radiometric non-linearities in the system to rectify systematic errors in the Phase Map for the surface/object being scanned. Section 7 explains our method in detail.

3D scanning phase: During this phase, we project sinusoidal coded patterns on an unknown object/surface. We compute its Phase Map, (optionally) rectify it for radiometric errors and estimate $3 \mathrm{D}$ point cloud using cross-ratios, as explained in Sect. 5.

\section{View-space codification}

Before we present our proposed calibration approach, we first discuss our approach to view-space codification, i.e. Sinusoidal Codification. As stated earlier, unlike in our method, 


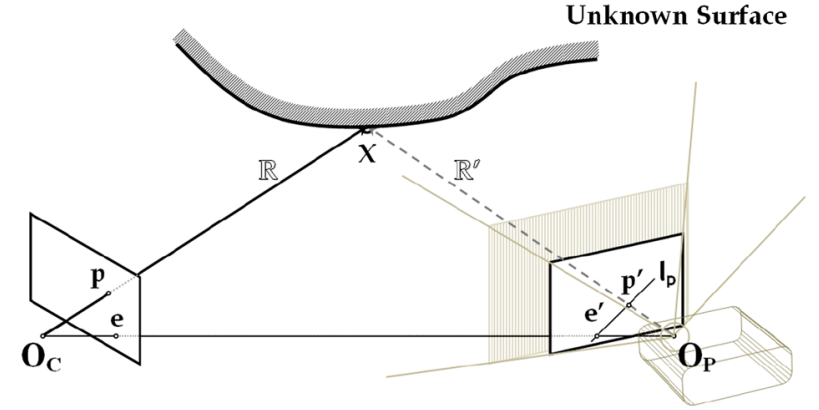

View 1 (Camera)

View 2 (Projector)

Fig. 1 Epipolar geometry of a projector-camera pair

sinusoidal coded patterns may be used to generate Reference maps during the Calibration phase. However, we use sinusoidal coded patterns only for solving the correspondence problem during $3 D$ Scanning phase. An understanding of view-space codification helps to understand the role of Reference Maps in our proposed approach for implicit calibration and $3 \mathrm{D}$ estimation.

To achieve dense reconstruction, we use view-space codification which allows us to uniquely identify each light ray emanating from the projector and determine its reflected image in the camera plane. While our method for geometric calibration and 3D estimation will be detailed in Sect. 5, here we note that the relative geometry of a projector-camera pair is identical to that of two cameras, see Fig. 1. Therefore, in the following discussion on view-space codification, we treat the projector as the second view. Structured-light systems address the problem of sparse correspondences under ambient lighting by illuminating the object by a known light pattern thereby enabling us to associate a unique codeword to every scene point illuminated by the projector. Further, the same codeword can be observed (i.e. decoded) from the images of the scene and we can associate points in the projector plane with points in the image plane if they share a unique codeword. Thus, structured-light systems convert the difficult correspondence problem into a simple codeword matching problem resulting in a dense 3D surface representation. For static scenes, temporal codification schemes are preferred since they encode each projector pixel independently. Throughout this paper, we use the temporal scheme of sinusoidal codification which is given below. The reader is also referred to [17] for a discussion of various pattern codification schemes.

In Fig. 1, let the projector ray passing through projector pixel $\mathbf{p}^{\prime}$ be reflected at the surface point $\mathbf{X}$ and be imaged at the camera pixel $\mathbf{p}$. Let the $x$-coordinate of $\mathbf{p}^{\prime}$ be denoted $x^{\prime}$. To codify the view-space, we use a sequence of $N$ equally phase-shifted vertical sinusoidal patterns where the $k$-th pattern $S^{k}, k=0 \ldots(N-1)$, is
$S^{k}\left(\mathbf{p}^{\prime}\right)=A+B \sin \left(\frac{2 \pi k}{N}+\Phi\left(\mathbf{p}^{\prime}\right)\right)$.

Here, $\Phi\left(\mathbf{p}^{\prime}\right)=\frac{2 \pi x^{\prime}}{C_{\Phi}}$, with $x^{\prime}$ being the $x$-component of $\mathbf{p}^{\prime}$ and $C_{\Phi}$ being the period of the sinusoid used. Also, $A$ is an offset term used to ensure positive gray levels and $B$ is the sinusoidal amplitude. The key point of such sinusoidal codification is that the phase value $\Phi\left(\mathbf{p}^{\prime}\right)$ uniquely codifies each column of pixels in the projector plane and this phase can be recovered from the sequence of images observed by the camera. For the projected images $S^{k}$, we denote the corresponding observed camera images as $I^{k}$. As the camera pixel $\mathbf{p}$ corresponds with projector pixel $\mathbf{p}^{\prime}$, the phase associated with the sequence $I^{k}(\mathbf{p})$ should also be $\Phi\left(\mathbf{p}^{\prime}\right)$. The actual decoded phase at $\mathbf{p}$ is given by

$\hat{\Theta}(\mathbf{p})=\tan ^{-1}\left(\frac{\sum_{k} I^{k}(\mathbf{p}) \cos (2 \pi k / N)}{\sum_{k} I^{k}(\mathbf{p}) \sin (2 \pi k / N)}\right)$.

Since the estimated phase is always wrapped to the range $[0,2 \pi)$, the estimated phase is given by,

$\hat{\Theta}(\mathbf{p})=\Phi\left(\mathbf{p}^{\prime}\right)-2 \pi\left\lfloor\Phi\left(\mathbf{p}^{\prime}\right) / 2 \pi\right\rfloor$.

Typically, $C_{\Phi}$ is much smaller than the projector plane's width to avoid poor quantisation of sinusoidal patterns and we need to unwrap $\hat{\Theta}(\mathbf{p})$ into $\Theta(\mathbf{p})$ to match with $\Phi\left(\mathbf{p}^{\prime}\right)$. We use graycodes for phase unwrapping as explained in [17]. Each column of projector pixels is assigned a graycode that has an equivalent code of $\left\lfloor\Phi\left(\mathbf{p}^{\prime}\right) / 2 \pi\right\rfloor$ which is the same for all $\mathbf{p}^{\prime}$ in a given column. We project these graycode patterns sequentially along with the sinusoidal patterns. The camera images corresponding to the graycode patterns are binarised and stacked together to give a graycode, i.e. the value obtained by taking the pixel $\mathbf{p}$ in each of the binary graycode images. Using the equivalent binary codeword $\beta(\mathbf{p})$, the unwrapped phase value for a camera pixel $\mathbf{p}$ is obtained as

$\Theta(\mathbf{p})=\hat{\Theta}(\mathbf{p})+2 \pi \beta(\mathbf{p})$.

While using the projector as the second view, solving the correspondence problem becomes a trivial task. A decoded phase in the image plane indicates the matching location in the projector, i.e. given $\Theta(\mathbf{p})$ in Eq. (4), we look for projector pixel $\mathbf{p}^{\prime}$ such that $\Theta(\mathbf{p})=\Phi\left(\mathbf{p}^{\prime}\right)$. As we shall see later, in combination with knowledge of the geometry of the projector-camera pair, we can use this relationship to solve for the matching location. Since we use a known $\Phi\left(\mathbf{p}^{\prime}\right)$ for the projector, knowing the decoded phase map is the same as knowing a dense correspondence between the camera and projector pixels. Thus, in the remainder of this paper, when we refer to a phase map we implicitly refer to the known dense correspondence map between the camera and projector planes. 


\section{Proposed approach for geometric calibration and 3D estimation}

In this section, we describe our proposed method for geometric calibration and 3D estimation that utilises the projective geometry between two views. Throughout we shall use the pin-hole model to describe the geometry of the projector and camera, please see [18] for details, and use two-view projective geometric relationships to calibrate the projectorcamera pair and for 3D estimation. However, since the direction of light rays is reversed in a projector, we cannot directly observe points (pixels) on the projector plane requiring an indirect approach for its calibration. While the general phase map between projector and camera is arbitrary, when viewing a planar scene this relationship has a simple parametric form that can be exploited. Without loss of generality we attach a 3D reference frame to a camera that can be calibrated as described earlier. Let the projector undergo a rigid 3 D rotation $\mathbf{R}$ and 3D translation $\mathbf{t}$ with respect to this frame of reference. Furthermore, we denote the intrinsic calibration of the camera and the projector as $\mathbf{K}_{C}$ and $\mathbf{K}_{P}$, respectively, which are $3 \times 3$ upper triangular matrices. Therefore, the projection matrices for the camera and projector are,

$\mathbf{P}_{C}=\mathbf{K}_{C}\left[\mathbf{I}_{3 \times 3} \mid \mathbf{0}_{3 \times 1}\right]$,

$\mathbf{P}_{P}=\mathbf{K}_{P}\left[\mathbf{R}_{3 \times 3} \mid \mathbf{t}_{3 \times 1}\right]$.

For an unknown 3D point $\mathbf{X}=(X, Y, Z)$ with the projections at $\mathbf{p}=(x, y)$ in the first view (camera) and $\mathbf{p}^{\prime}=\left(x^{\prime}, y^{\prime}\right)$ in the second view (projector), we have

$\mathbf{p}=\left[\begin{array}{lll}x & y & 1\end{array}\right]^{T}=\lambda_{1} \mathbf{P}_{C}\left[\begin{array}{llll}X & Y & Z & 1\end{array}\right]^{T}$,

$\mathbf{p}^{\prime}=\left[\begin{array}{lll}x^{\prime} & y^{\prime} & 1\end{array}\right]^{T}=\lambda_{2} \mathbf{P}_{P}\left[\begin{array}{llll}X & Y & Z & 1\end{array}\right]^{T}$.

Here, $\lambda_{1}$ and $\lambda_{2}$ are unknown scale factors and [ $\left.\begin{array}{lll}x & y & 1\end{array}\right]$ is the homogeneous representation of $\mathbf{p}$.

For two views, given the homogeneous point $\mathbf{p}$ in the first view (camera) and its corresponding homogeneous point $\mathbf{p}^{\prime}$ in the second view (projector), we have the epipolar relationship, $\mathbf{p}^{\prime T} \mathbf{F p}=0$ where $\mathbf{F}$ is a $3 \times 3$ matrix known as the Fundamental Matrix which has a rank of 2 with only 7 degrees of freedom. The Fundamental Matrix is of the form $\mathbf{F}=\mathbf{K}_{P}^{-T}[\mathbf{t}]_{\times} \mathbf{R} \mathbf{K}_{C}^{-1}$ where [ ] $\times$ denotes the skewsymmetric form of a vector. Given a sufficient number of matched point pairs (p, $\left.\mathbf{p}^{\prime}\right), \mathbf{F}$ can be estimated, see [18].

\subsection{Homography induced by a plane}

While the epipolar relationship $\mathbf{p}^{\prime T} \mathbf{F p}=0$ is always satisfied, when the object being viewed is a plane (denoted by $\Pi$ in Fig. 2), every corresponding pair of image points (p, $\left.\mathbf{p}^{\prime}\right)$ of the observed plane also obeys the relationship $\mathbf{p}^{\prime}=\mathbf{H p}$,

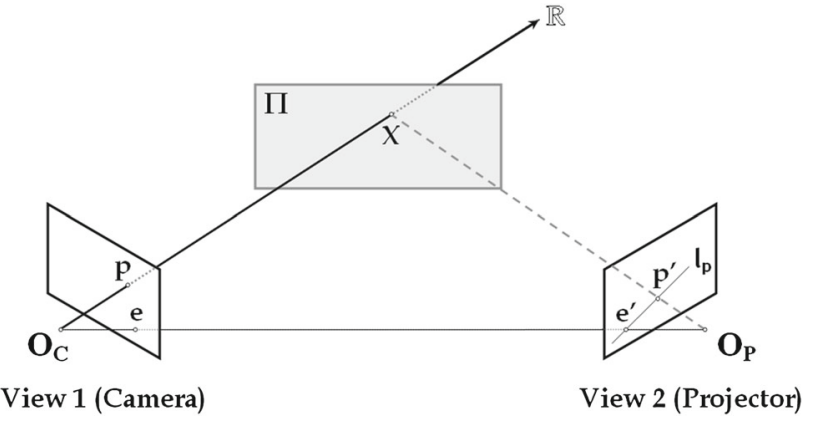

Fig. 2 The $3 D$ plane $\Pi$ induces a homography that relates the correspondences in the two views

where the equality is up to scale (i.e. projective) and the $3 \times 3$ matrix, $\mathbf{H}$ is known as the homography induced by the plane. The homography $\mathbf{H}$ has 8 degrees of freedom (nine elements of the matrix minus one for an overall scale) and can be accurately estimated given enough correspondences. Thus, while the reference phase map has a value associated with each pixel, it is in fact a low-dimensional mapping and can be fully described by a homography with only 8 degrees of freedom, i.e. for projector pixel $\mathbf{p}^{\prime}$, we should observe the same phase in the corresponding camera pixel $\mathbf{p}=\mathbf{H}^{-1} \mathbf{p}^{\prime}$, i.e. $\Phi\left(\mathbf{p}^{\prime}\right)=\Theta\left(\mathbf{H}^{-1} \mathbf{p}^{\prime}\right)$.

The other techniques in the literature that utilise reference planes $[7,16]$ fail to make use of this important observation. Consequently, they retain the entire measured phase map, i.e., a phase value at each camera pixel. The key contribution of our calibration technique is that for each reference plane used, we represent the phase map by a homography represented by eight parameters. This results in greatly improved accuracy of the phase map since the noise in individual observed phase values are averaged out in the homography estimation. Crucially, it should also be noted that the homography relationship holds for planes in general position. As a result, unlike some other methods, we do not rely on accurate placement and precisely controlled movement of the reference plane. Our reference plane can be placed arbitrarily and does not need any precisely guided mechanical devices for placement.

To calibrate the camera using the method in [20], we need to acquire a set of images of a reference calibration plane placed in different positions. For this purpose, we use a planar object onto which we paste the pattern in Fig. 3a. To compute the plane-induced homographies, we need to project a single fixed grid pattern (Fig. 3b) onto each reference plane and establish correspondences for the grid corner locations in the projector plane and their respective images in the camera plane. These correspondences are then used to solve for the homography induced between the projector and the camera. All such correspondences (i.e. for each reference plane) satisfy the epipolar geometry as well. We thus use all of them together, as a large set of reliable correspondences, to esti- 


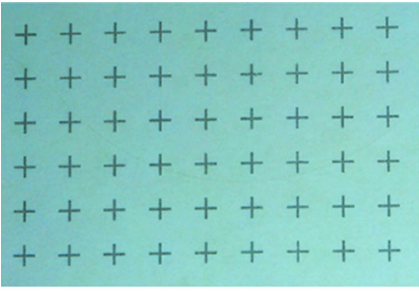

(a)

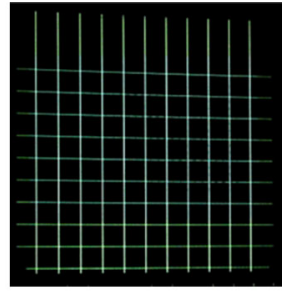

(b)
Fig. 3 a Camera image of calibration pattern with ' + ' marks, $\mathbf{b}$ camera image of the grid pattern projected onto reference plane to compute homographies

mate the fundamental matrix accurately using a robust $M A P$ $S A C$ estimator [21]. It may be noted that to reliably establish the homography induced by the plane, we only need a few matched points in the projector and camera planes. As a result, the grid pattern of Fig. 3b suffices and we do not need to use the full sinusoidal codification to establish a complete reference phase map that matches all points in the camera plane to those in the projector plane. In other words, during the calibration step we can use a simple grid image pattern and only need the sinusoidal codification patterns during the scanning step.

To simplify the calibration step, we can use the same calibration reference plane placed in different locations for acquiring both the image of the calibration marker pattern (Fig. 3a) for camera calibration and the image of the grid pattern projected onto it (Fig. 3b) for computing the homography induced by the plane. Our method thus acquires images for projector and system calibration simultaneously with the images for camera calibration, see [1]. When we project a grid pattern onto a given reference plane and locate their images in the camera plane, the 3D-2D projection induced is also a homography, i.e. there exists a linear projective relationship between every grid corner image in the camera and its corresponding 3D point location on the reference plane. In fact, given this 3D-2D homography and calibration, for every pixel in the camera plane we can easily determine the location of the intersection of the reference plane with the ray through the camera centre and the given pixel [20]. This allows us to estimate the 3D location of a point on a surface in the view-space via a well-known projective invariant, i.e., the cross-ratio.

\subsection{Using the cross-ratio}

For four collinear points $\mathbf{p}_{1}, \mathbf{p}_{2}, \mathbf{p}_{3}, \mathbf{p}_{4}$, their cross-ratio is defined as

$C R\left(\mathbf{p}_{1}, \mathbf{p}_{2}, \mathbf{p}_{3}, \mathbf{p}_{4}\right)=\frac{\left\|\left(\mathbf{p}_{1}-\mathbf{p}_{3}\right)\left(\mathbf{p}_{2}-\mathbf{p}_{4}\right)\right\|}{\left\|\left(\mathbf{p}_{2}-\mathbf{p}_{3}\right)\left(\mathbf{p}_{1}-\mathbf{p}_{4}\right)\right\|}$

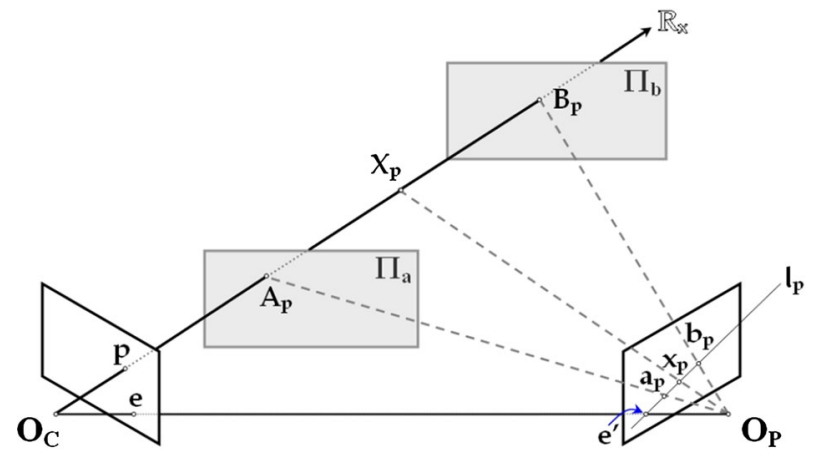

View 1 (Camera)

View 2 (Projector)

Fig. 4 Using reference planes for estimating 3D point locations using cross-ratios

It is a fundamental result in projective geometry that the cross-ratio is invariant to projective transformations (see Sec 2.5 of [18]). Therefore, the cross-ratio measured in a projective frame of reference can be directly related to the crossratio measured in a Euclidean frame of reference. This allows us to use the cross-ratios measured in the projector plane to solve for 3D depth, given that the rays falling onto a camera's image plane are calibrated in a Euclidean sense [7]. Let $\mathbf{X}_{p}$ be an unknown 3D point that is imaged at a camera pixel location $\mathbf{p}$ and let its back-projected ray be denoted, $R_{x}$, see Fig. 4. We note that the camera centre $O_{C}$ and the two points on reference planes $\Pi_{A}$ and $\Pi_{B}$, i.e. $A_{p}$ and $B_{p}$ are all collinear as they lie on the back-projected 3D ray, $R_{x}$. Here, we note that there exists a projective transformation that maps the ray $R_{x}$ onto the projector plane generating the epipolar line $\left(l_{p}\right)$ corresponding to pixel $\mathbf{p}$ in the camera plane. The corresponding images on this epipolar line of the collinear points $O_{C}, A_{p}, X_{p}$, and $B_{p}$ are $e^{\prime}, a_{p}, x_{p}$ and $b_{p}$, respectively, where $e^{\prime}$ is the epipole in the projector plane. Since this is a projective transformation applied to a line, we have the invariance of the cross-ratio, i.e.

$C R\left(O_{C}, A_{p}, X_{p}, B_{p}\right)=C R\left(e, a_{p}, x_{p}, b_{p}\right)$.

Furthermore, an orthogonal projection from $l_{p}$ to the column axis maps the points to their column indices and is itself a projective transformation. Thus,

$C R\left(e^{\prime}, a_{p}, x_{p}, b_{p}\right)=C R\left(c_{e^{\prime}}, c_{a_{p}}, c_{x_{p}}, c_{b_{p}}\right)$.

Similarly, the cross-ratio for any four given points on the ray $R_{x}$ is equal to the cross-ratio for their coordinates along the Cartesian $z$-axis which results in,

$C R\left(Z_{O_{C}}, Z_{A_{p}}, Z_{X_{p}}, Z_{B_{p}}\right)=C R\left(O_{C}, A_{p}, X_{p}, B_{p}\right)$.

Using Eqs. (10), (11) and (12), we get

$C R\left(Z_{O_{C}}, Z_{A_{p}}, Z_{X_{p}}, Z_{B_{p}}\right)=C R\left(c_{e^{\prime}}, c_{a_{p}}, c_{x_{p}}, c_{b_{p}}\right)$. 
Now, locations $c_{e^{\prime}}, c_{a_{p}}, c_{x_{p}}, c_{b_{p}}$ are nothing but the $x$ coordinates (i.e. column locations) of $e^{\prime}, a_{p}, x_{p}, b_{p}$, respectively, in the projector plane. $c_{a_{p}}$ and $c_{b_{p}}$ can be estimated using homographies induced by planes $\Pi_{A}$ and $\Pi_{B}$, respectively. $e^{\prime}$ (and thus $c_{e^{\prime}}$ ) can be obtained from the estimated Fundamental matrix $\mathbf{F}$ and $c_{x_{p}}$ is estimated by decoding sinusoidal phase at camera pixel location $\mathbf{p}$ for the unknown point $\mathbf{X}_{p}$. Thus, using estimated values for $c_{e^{\prime}}, c_{a_{p}}, c_{x_{p}}$ and $c_{b_{p}}$, the r.h.s for Eq. (13) can be calculated. Again, the camera centre $O_{C}$ is at the origin of our frame of reference and the equations of the reference planes $\Pi_{A}$ and $\Pi_{B}$ can be obtained from the 3D-2D homographies between the camera and each of the corresponding reference planes (see [20] for details). Given the plane equations for $\Pi_{A}$ and $\Pi_{B}$, we can obtain the 3D coordinates of $A_{p}$ and $B_{p}$ by intersecting the backprojected ray (for pixel p) with these planes. Since the other $Z$-coordinates in the 1.h.s for Eq. (13) are now estimated, the only unknown quantity $Z_{X_{p}}$ can be solved for using Eq. (13).

\subsection{Advantages of the proposed approach}

Our approach to calibration and 3D estimation presented above has considerable advantages over other existing methods. The most important advantage of our method is that it represents the entire phase map by an eight-parameter homography. Since we only need to solve for the eight degrees of freedom, the entire phase map can be accurately estimated by projecting a single grid pattern on the reference plane with an arbitrary placement. This makes our calibration stage much faster than in $[5-7,16]$ which need to project an entire pattern sequence on each reference plane as the phase map has to be estimated for every pixel. This process is considerably slow for $[6,7]$ which require precise control for parallel placement of reference planes. In addition, since [6,7] do not impose the homography constraint, they cannot mitigate localised phase map errors due to noise, optical blur, etc. In contrast, since in our method we fit an eight-parameter homography using a large number of correspondences, the individual errors are averaged out. Consequently, as we will demonstrate in Sect. 6.2, our method achieves sub-millimetre accuracy.

Methods based on the cross-ratio defined by four collinear points require that the calibration be carried out for three reference planes. In our case, apart from the projector-camera correspondences induced by a plane satisfying a homography for each reference plane, all these correspondences also collectively satisfy the epipolar geometry between the projector and camera. As a result, two reference planes are sufficient since we get the third cross-ratio point for 'free' as the origin of the camera-based frame of reference and the epipole in the projector plane. Given the large number of reliable correspondences generated using two reference planes, the epipole can be estimated accurately. Furthermore, in our method, codification along one dimension is sufficient for 3D-point estimation. This halves the image acquisition time during scanning.

Our homography model provides a specific advantage in terms of the field of view that may be scanned. Unlike methods that use complete reference phase maps, in our case even if the calibration is carried out using a plane that only spans a small area of the camera field of view, the estimated homography applies to the entire field of view, i.e., during the scanning process we can reconstruct the entire view-space imaged by the camera. This simplifies the task of placement of reference planes during calibration and it is particularly advantageous when scanning a large object where it might be difficult to place reference planes in a manner that they span the entire field of view of the camera. A final significant advantage that may be mentioned is that since our geometric relationship is parametric, we can directly achieve subpixel correspondence localisation and do not need to carry out expensive interpolated searches as in $[6,7]$.

\section{Experiments and results}

In this section, we detail a series of qualitative and quantitative experiments that demonstrate the accuracy of our method. Throughout, we used a Canon S5IS digital camera and an NEC NP400 LCD projector set at resolutions of $2048 \times 1536$ and $1024 \times 768$ pixels, respectively. Each homography was estimated using upto 121 grid point correspondences (see Fig. 3b), and the Fundamental Matrix was estimated using MAPSAC [22] using all such correspondences. While we identified shadow pixels and smoothed the phase signal, in the case of quantitative comparisons, we did not smooth the decoded phase signal to allow for a true comparison of the performance of different methods. Since in these experiments we are interested in evaluating our geometric calibration, we used $N=32$ phase-shifted sinusoidal patterns for phase estimation. Using a large number of patterns allowed us to remove the effect of radiometric non-linearities that are addressed in Sect. 8 where we provide results with as few as $N=4$ phase-shifted patterns.

\subsection{Qualitative evaluation}

The first set of results we detail here constitute a qualitative evaluation of our method. We scanned objects with varying degrees of surface complexity and the results were visually examined. Figure 5 shows scan results for simple surfaces that appear to be accurately recovered. Figure 6 shows results for an object with surfaces of moderate complexity, a clay figurine of the Hindu god, Ganesh. Fine details on the trunk, head and limbs are distinctly noticeable in the surface rendering. We also scanned another object with a significantly high 


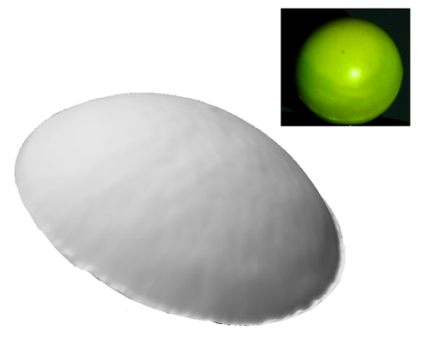

(a)

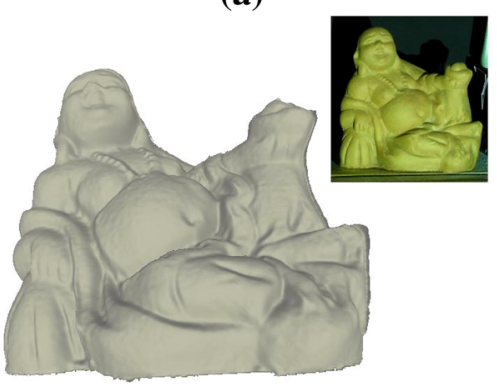

(c)
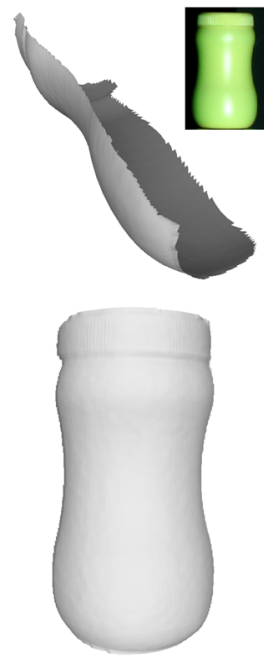

(b)

Fig. 5 Scan results for simple surfaces (with images in the insets): a sphere, $\mathbf{b}$ surface of revolution, and $\mathbf{c}$ a Buddha figurine

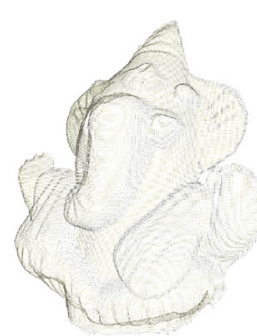

(a)

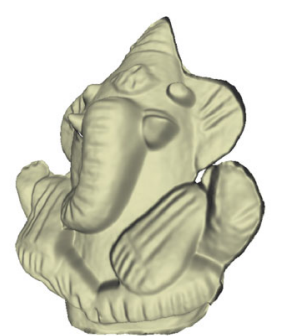

(b)

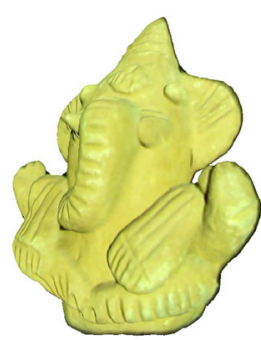

(c)

Fig. 6 Scan results for an object $(70 \mathrm{~mm}$ by $90 \mathrm{~mm})$ with moderate surface complexity, a clay figurine of the Hindu god, Ganesh. a Estimated Point Cloud, b estimated Mesh, and $\mathbf{c}$ texture-mapped Mesh

degree of surface complexity, a clay idol with five heads, i.e. the panchamukhi Hanuman. The reconstruction results are shown in Fig. 7. As can be seen, details of the irises in the eyes, nostrils, features on the hand and ornamental details on the chest are accurately reconstructed. We also note that even finer details like etching marks on legs are clearly visible. These details are shown in Fig. 7c with close-up views of selected regions of the scan.

We also tested our scanning approach for generating a complete model using eight different scan views of a Buddha figurine as shown in Fig. 8a. These individual scans were aligned and merged using standard methods in Meshlab [23]. As can be seen in Fig. 8b, due to the accuracy of the individual scans, the aligned scans are merged seamlessly into a single surface.

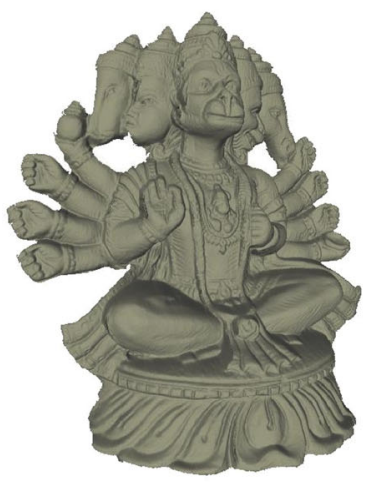

(a) Lateral-view

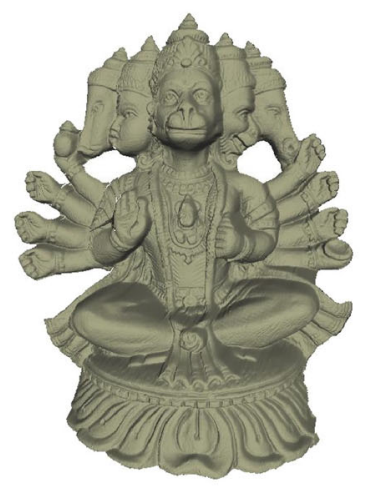

(b) Front-view

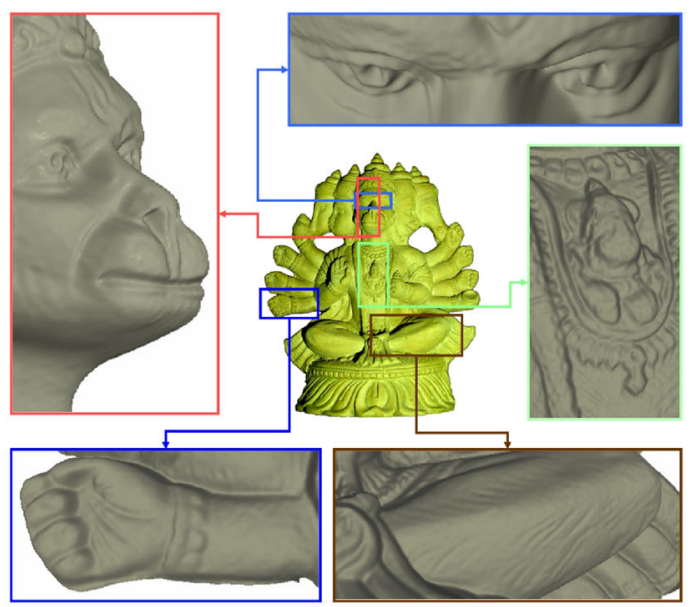

(c) Close-up views showing details

Fig. 7 Scan results for a complex object, a clay idol of the panchamukhi Hanuman that is $250 \mathrm{~mm}$ wide and $280 \mathrm{~mm}$ high

\subsection{Quantitative evaluation}

To characterise the accuracy of our approach, we also carried out quantitative experiments by scanning a plane placed at an arbitrary orientation. As shown in Table 1, the root mean squared (RMS) plane fitting error for the recovered plane (Plane 1) characterises the impact of different factors during calibration. Column (a) indicates the results using three complete 'raw' reference phase maps, i.e. we do not estimate homographies using these maps. Column (b) indicates the results when homographies are estimated for the three reference maps. Note that in both (a) and (b), we do not compute the epipole and instead use three reference maps. For columns (d), (e) and (f), multiple reference planes were used (up to 5), where we average multiple 3D estimates that use two reference planes and the epipole to compute crossratios. Compared to the basic method in column (a), using the homographies improved scanning accuracy by about $12 \%$ which is very significant. This is strong evidence that fitting a low-dimensional homography is highly advantageous as the noise in the raw reference map is averaged out, leading to 


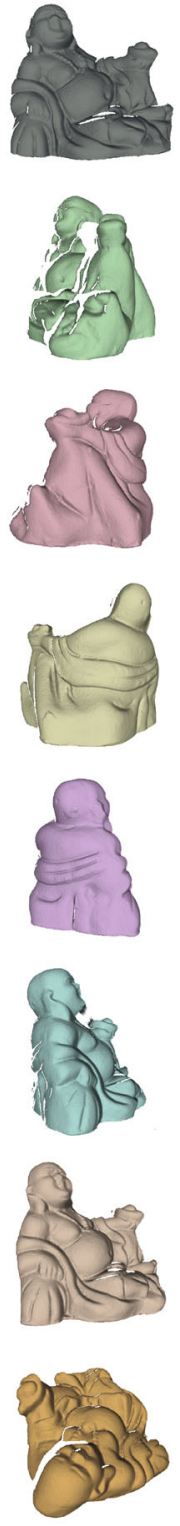

(a) Individual Scans
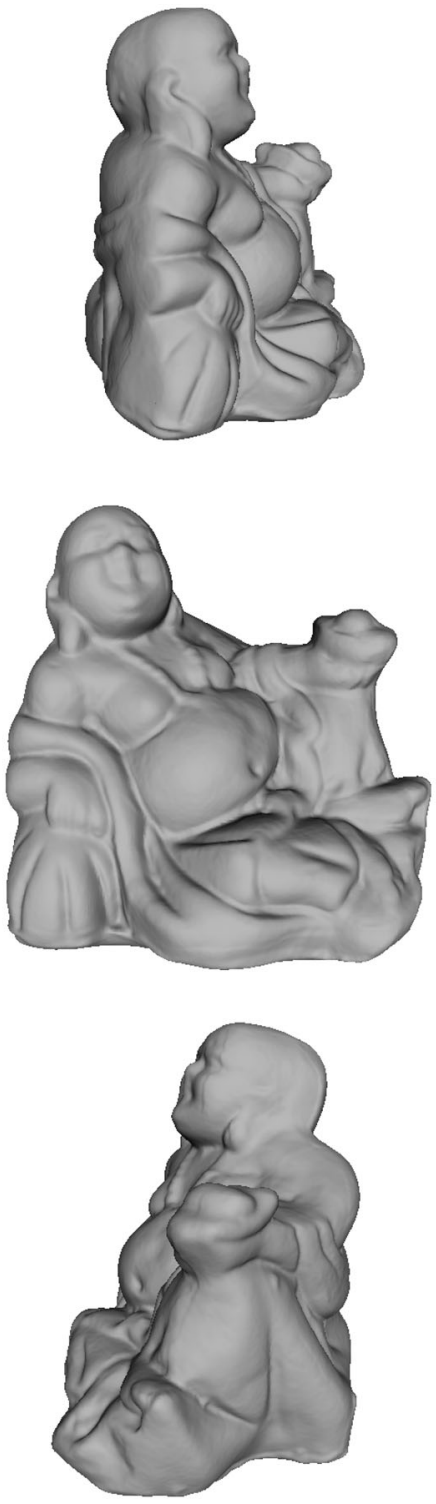

(b) Merged model (Different views)

Fig. 8 Generated complete model for a Buddha figurine

improved accuracy. Table 1 also shows error measures for the reconstruction of the unknown plane in three other positions. These reconstructions were performed using four reference planes along with the fundamental matrix. The individual estimates using two reference planes at a time are averaged. The RMS error is observed to be on the order of one-tenth of a millimetre for each of these scans. We reiterate that we did not use any smoothing at either the pre-processing or the post-processing stage for the plane fitting evaluation.

In another experiment, we evaluated the accuracy of 3D estimation for the panchamukhi Hanuman model described earlier. We physically measured a set of five distances using Vernier Callipers with a precision of $0.05 \mathrm{~mm}$ (see Fig. 9). To account for human error, each distance was measured ten times and its average value was used for comparison. Measured distances spanned across all three Euclidean dimensions. The same set of distances was estimated from the reconstructed model by carefully locating the end points and the comparison is presented in Table 2. The absolute differences in the estimated distances are observed to be on the order of $0.1 \mathrm{~mm}$ providing further evidence for the high accuracy of our reconstructions.

To study the impact of inaccuracies in calibrating the system using our approach, we performed multiple reconstructions of the same surface using different pairs of reference planes and then estimating the variance of the depth estimates. If we use $n$ reference planes, we have as many as $\left(\begin{array}{l}n \\ 2\end{array}\right)=\frac{n(n-1)}{2}$ reference pairs, each of which gives us a $3 \mathrm{D}$ estimate. This variance is a measure of the uncertainty of our individual 3D estimates and arises due to (a) inaccuracies in calibrating the individual reference planes and (b) inaccuracies in homography estimation. The computed variance statistics for Ganesh and Hanuman idols using different pairs from a set of $n=5$ reference planes are presented in Table 3. The median value for standard deviation for each of these scans is about half a millimetre, implying that our method gives consistently good results independent of the placement of the reference planes during calibration.

Table 1 Error measures for planar surfaces with different methods/conditions

\begin{tabular}{|c|c|c|c|c|c|c|c|c|c|}
\hline & \multicolumn{6}{|l|}{ Plane 1} & \multirow[t]{2}{*}{ Plane 2} & \multirow[t]{2}{*}{ Plane 3} & \multirow[t]{2}{*}{ Plane 4} \\
\hline & (a) & (b) & (c) & (d) & (e) & (f) & & & \\
\hline Mean depth & \multicolumn{6}{|c|}{1100.50} & 1191.70 & 1004.32 & 959.12 \\
\hline Median |Error| & 0.0988 & 0.0979 & 0.0974 & 0.0980 & 0.0979 & 0.0977 & 0.073 & 0.047 & 0.085 \\
\hline Max |Error| & 0.6593 & 0.4463 & 0.4444 & 0.4470 & 0.4482 & 0.4468 & 0.783 & 0.323 & 0.592 \\
\hline RMS error & 0.1426 & 0.1266 & 0.1264 & 0.1262 & 0.1262 & 0.1259 & 0.110 & 0.071 & 0.125 \\
\hline
\end{tabular}

For Plane 1, (a) represents basic cross-ratio method using three complete reference phase maps (no homographies). (b) is for method with three reference maps represented using homographies; (c)-(f) used 2-5 reference map homographies along with epipolar geometry. For Planes 2-4 four reference map homographies along with epipolar geometry were used. All units are in $(\mathrm{mm})$ 
Table 2 Distances measured physically and from reconstructed model for Hanuman idol are shown along with absolute errors

\begin{tabular}{llllll}
\hline Source & \multicolumn{5}{l}{ Distance measured } \\
\cline { 2 - 6 } & $D_{1}$ & $D_{2}$ & $D_{3}$ & $D_{4}$ & \multicolumn{1}{l}{$D_{5}$} \\
\hline Physical Object & 128.515 & 168.415 & 161.540 & 69.565 & 137.290 \\
$\begin{array}{c}\text { Reconstructed } \\
\text { Model }\end{array}$ & 128.625 & 168.386 & 161.421 & 69.407 & 137.312 \\
IError| & 0.110 & 0.029 & 0.119 & 0.158 & 0.022
\end{tabular}

$D_{1}$ is from rightmost head's eye to leftmost head's eye, $D_{2}$ is from left eye to the tip of rightmost hand's thumb, $D_{3}$ is from tip of the head mark to centre of the flower at base, $D_{4}$ is from a key point on abdominal ornament to tip of the rightmost groove at base, $D_{5}$ is from centre of the necklace to tip of the rightmost groove at base (see Fig. 9). Average depth for the surface is $1091.79 \mathrm{~mm}$. All units below are in $(\mathrm{mm})$

Table 3 Error statistics for non-parametric surfaces

\begin{tabular}{lll}
\hline Error measure $(\mathrm{mm})$ & Ganesh idol & Hanuman idol \\
\hline Minimum $\sigma$ & 0.277 & 0.084 \\
Maximum $\sigma$ & 1.111 & 1.334 \\
Median of $\sigma$ & 0.546 & 0.439
\end{tabular}

Point wise standard deviation id computed from multiple estimates for each surface using five reference planes

We remark here that although [9] also uses projective geometric relationships like us, the qualitative accuracy of our results in Figs. 5, 6, 7 and 8 is visibly much higher than the 3D reconstruction presented in [9]. Also, accuracy of our reconstruction of planar surfaces, as presented in Table 1, is comparable to the accuracy of Moreno and Taubin's method, as presented in Table III and Figure 8 in [8]. We note again that our approach avoids the computational complexities of their method pertaining to non-linear minimization for projector calibration, computation of numerous local homographies and decoding graycode maps for each calibration plane position. We also avoid the acquisition of a multitude of calibration plane images when the calibration plane is projected with a series of coded patterns for each position, specifically to calibrate the projector.

Having demonstrated the effectiveness of our geometric calibration, we now describe the effects of radiometric nonlinearities and develop our solution for correcting the radiometric and geometric non-linearities present.

\section{Radiometric correction}

Since phase-shifted sinusoidal patterns are an example of temporal codification [17], they have the advantage of encoding each projector pixel independently. This avoids the difficulties associated with spatial encoding schemes where neighbourhood regions get distorted due to perspective pro-

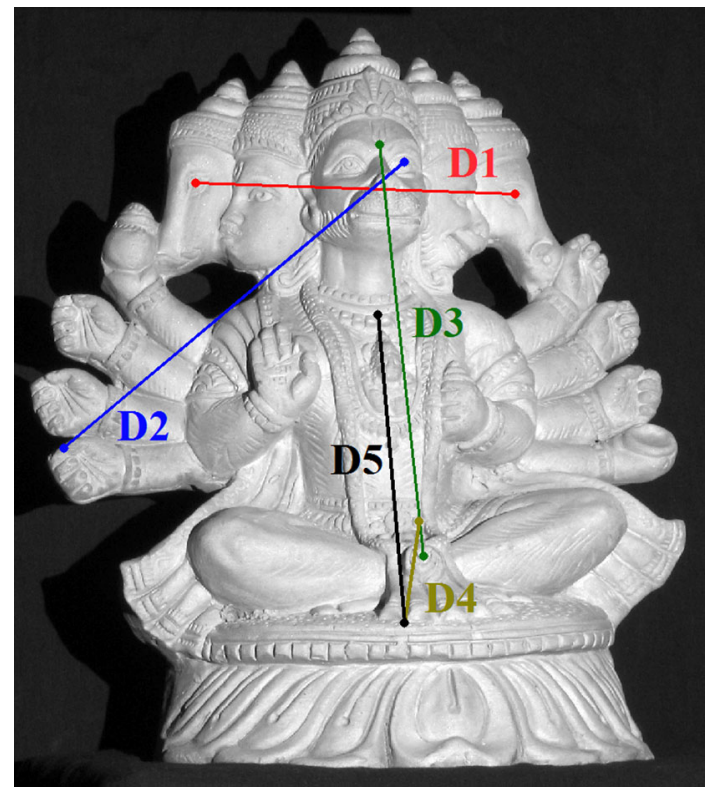

Fig. 9 Hanuman figurine with different distances marked. See Table 2 and text for details

jection and/or occlusion [17]. Furthermore, sinusoidal codification requires fewer patterns than other temporal schemes to encode the view-space. However, most digital projectors have a non-linear radiometric behaviour known as gamma distortion which introduces errors in the output intensities which lead to errors in the recovered reference phase maps and consequently in the correspondences. Other factors such as camera aperture size, exposure time and optical blur also contribute to the overall distortion of the intensities measured by the camera. Under gamma distortion, the output luminance $L_{\mathrm{op}}$ of a digital projector is not linearly related to input gray-level $I_{\text {ip }}$ but obeys the form,

$L_{\mathrm{op}}=L_{\max }\left(\frac{I_{\mathrm{ip}}}{I_{\mathrm{max}}}\right)^{\gamma}$.

Here, $I_{\max }$ is the maximum possible input gray level, $L_{\max }$ is the maximum possible output luminance and $\gamma$ is the gamma distortion which is often greater than 1 . As we can see in Eq. 2, the accuracy of the estimated phase $\hat{\Phi}(\mathbf{p})$ depends on the correctness of the intensities $I^{k}(\mathbf{p})$ observed by the camera. In the presence of gamma distortion, an error is introduced into the recovered phase which translates into an error in the estimated 3D locations in the point cloud recovered. When the number of encoding phase-shifted sinusoids used $(N)$ is large, the radiometric error introduced into the individual intensities $I_{k}$ tends to cancel out and the overall effect of gamma distortion is negligible. However, this implies that the data acquisition during the scanning process takes longer since many phase-shifted sinusoids need to be projected and imaged by the camera. When a lower number of phase-shifted sinusoids are used, while the acquisition time is reduced, the 
gamma distortion becomes pronounced and manifests as an artefact of a wave-like structure superimposed on the recovered 3D object shape. See Fig. 12a for an example of the recovered shape of a planar object that demonstrates this effect. Apart from such radiometric distortion, the overall calibration is also affected by geometric errors like radial distortion, errors in the estimation of the induced homography and noise in the intensity observations. Thus, in all, the observed reference phase map can be described as being composed of a linear projective map given by the homography which is distorted by the above-mentioned radiometric and geometric non-linearities.

To model the non-linear radiometric and residual geometric errors that present themselves as systematic variations in the reference phase map, we need a model that can capture smooth variations while avoiding over-fitting. This requirement is well satisfied by a cubic spline as it fits the data using control points while ensuring that the first and second derivatives of the fitted function is continuous over the entire domain. In the following, we use cubic splines to model the systematic variation. By placing a reference plane at an arbitrary orientation we obtain a raw phase map $\Phi_{\text {orig }}\left(\mathbf{p}^{\prime}\right)$ in the projected images. Subsequently, we decode the phase in the image plane to get the corresponding reference phase map. Now, using our estimated homography $\mathbf{H}$, we map the camera phase map onto the projector plane which we denote $\Phi_{\text {est }}\left(\mathbf{p}^{\prime}\right)$. Here, we use enough observations to estimate $\mathbf{H}$ independent of the non-linearities.

The resultant difference $\Phi_{\text {err }}=\Phi_{\text {orig }}-\Phi_{\text {est }}$ represents the error introduced by radiometric distortion and geometric errors. Unlike the radiometric errors which are periodic, the geometric errors (largely due to radial distortion) are aperiodic and exhibit a slow variation across the projector's columns. To eliminate this geometric error in $\Phi_{\text {est }}$ we use a moving average filter with a window size equal to that of a projected sinusoid's period, i.e. $C_{\Phi}$. Let us denote this moving average estimate of geometric error as $\Phi_{\mathrm{MA}}$. Finally, we define the residual error map as $\Phi_{\mathrm{re}}=\Phi_{\mathrm{orig}}-\Phi_{\mathrm{est}}-\Phi_{\mathrm{MA}}$. To model the errors induced by radiometric distortion, we wrap $\Phi_{\text {re }}$ over the phase period of $2 \pi$. Using this wrapped residual error map, we define control points that are uniformly distributed (in our case, at an empirically defined interval of $0.03 \pi$ ) and a cubic spline function is then estimated to pass through these control points. Figure 10 illustrates the spline fitting for a real-world reference plane. This estimated spline function provides a direct mapping between an estimated phase value and the expected systematic error due to radiometric distortion. During 3D estimation for an unknown surface, we simply subtract the expected systematic error given by the fitted cubic spline function from the estimated phase value. This approach has the ability to significantly reduce the effect of radiometric distortion that would otherwise be quite pronounced in the case

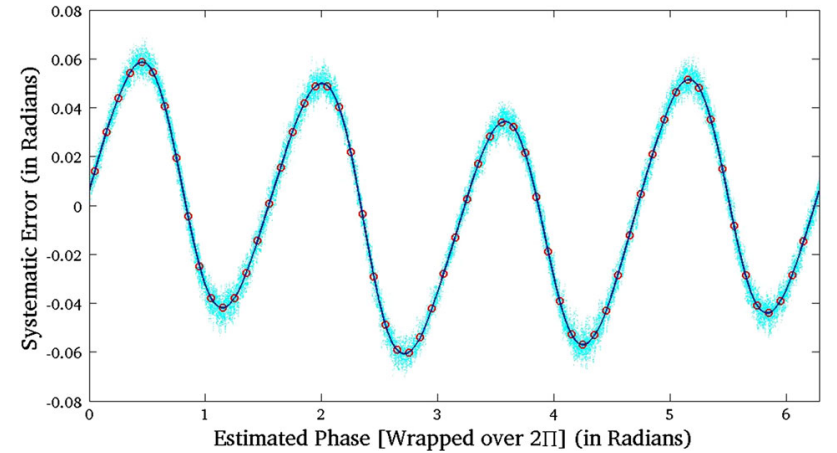

Fig. 10 Fitting cubic spline on systematic errors for a real-world reference plane

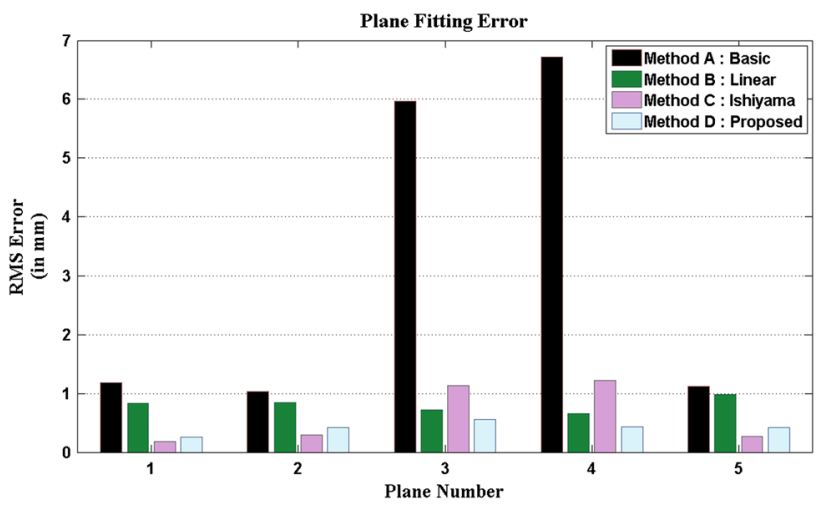

Fig. 11 Plane fitting error

when we use a few phase-shifted sinusoids in the scanning process.

\section{Radiometric correction experiments}

In this section, we describe the experiments carried out to evaluate our approach to radiometric correction. For this evaluation, we used the cross-ratio based 3D estimation using homographies induced by a set of three reference planes. Thus, estimating the Fundamental Matrix was avoided. As mentioned earlier, to evaluate the geometric calibration approach we used a large number of phase shifts $(N=32)$ which also provides a baseline for evaluating our radiometric correction method. In the experiments of this section, we used $N=4$ phase shifts. Using such a low number of phase shifts results in a significant impact of radiometric non-linearities which are corrected for using our radiometric correction approach.

In all, we used four different methods for quantitative comparisons. Method A is a basic system that uses three complete reference phase maps (i.e. without using homographies) and the basic cross-ratio approach for reconstruction. In contrast, Method B uses the estimated homographies induced by 
three reference planes along with cross-ratios for 3D reconstruction. Method $C$ is our implementation of the approach proposed in [7] that is presented for comparison with our approach. Finally, Method $D$ uses our proposed algorithm to rectify the phase map, i.e. Method $D$ is our radiometric correction applied to the phase map generated by Method $B$. Five planar surfaces were reconstructed using all the four methods. The same set of images of the target planes and ref-

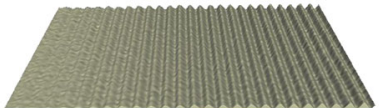

(a) Method A : Basic

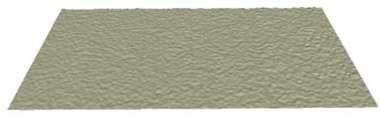

(c) Method C : Method of [7]

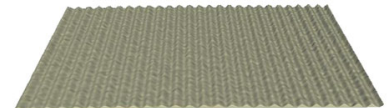

(b) Method B Homography-based

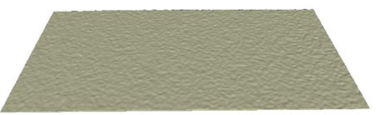

(d) Method D : Proposed Correction Method

Fig. 12 Plane reconstructed using different methods

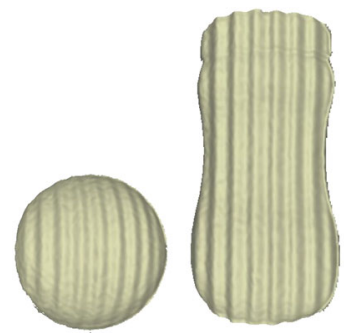

(a) Method B : Using homographies for phase maps erence planes was used by all the methods. Decoded phase maps for all target and reference planes were processed using the same low-pass Gaussian filter in all methods. For each reconstructed plane, a parametric plane was fitted and the RMS error for the different methods are shown in Fig. 11. For all cases, Method A shows a high amount of errors since it does not use any parametric model for calibration. Method $B$ improves upon Method A by fitting a homography model for representation of reference phase maps. However, it does not model the radiometric non-linearities present due to the low number of phase-shifted patterns used. Method $C$ of [7] greatly improves the results by modelling non-linearities using a method to compute cross-ratios along an arbitrary line in the one of the reference phase maps instead of projector's display plane. Our proposed method, i.e. Method D gives results that are comparable or better than that of Method $C$. In particular, for planes 3 and 4 where there are high errors in the target plane's phase estimation (due to the acute orientation of the reference plane), our approach does better by avoiding the interpolation errors that are present in the approach of [7] in Method C. Figure 12 shows the first plane in our quantitative evaluation as reconstructed by all four methods. Clearly, it can be seen by comparing Fig. 12a, d that our approach removes systematic artefacts effectively.

We also scanned some objects using method Method B and our radiometric correction proposal Method $D$ and the results are presented in Fig. 13. In addition, the panchmukhi Hanuman idol of Fig. 7 was reconstructed using Method $B$ with $N=32$ and $N=4$ phase shifts and the corresponding results are shown in Fig. 14a, b, respectively. Using the same set of images for $N=4$ phase shifts, we show the results using our proposed approach, i.e. Method D in Fig. 14c. Our approach to radiometric correction can be seen to effectively remove systematic visual artefacts that arise due to the use of

Fig. 13 Reconstruction of geometric surfaces

(b) Method D : Method B + Proposed spline modelling

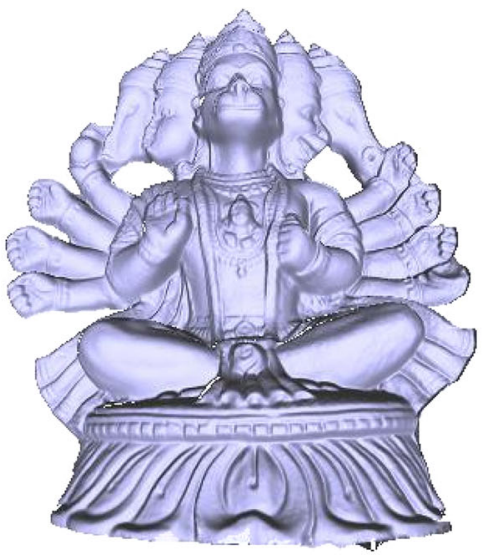

(a) Method B for $N=32$

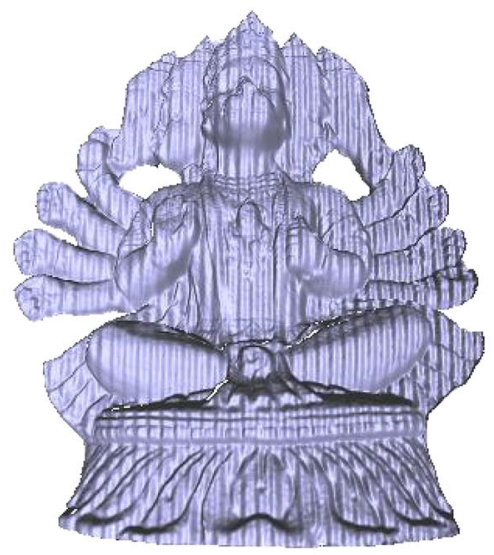

(b) Method B for $N=4$

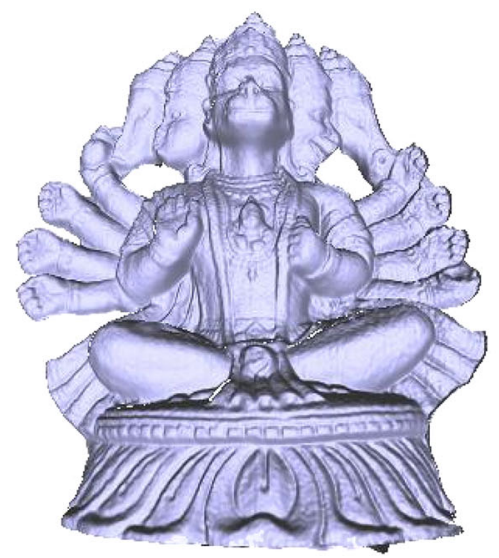

(c) Method D (Proposed method) : $N=4$

Fig. 14 Reconstruction of the panchmukhi Hanuman idol. Notice that in $\mathbf{c}$ our radiometric correction method can effectively correct for the errors observed in b 
only four phase shifts. Thus, our results adequately demonstrate that our radiometric correction approach can deal with significant distortion effects.

\section{Conclusion}

In this paper, we have presented a new approach to solve for geometric calibration and 3D estimation in structured-light scanners. By exploiting the homography induced by a plane in the calibration step, we developed a low-dimensional parametric representation of the phase map between the camera and projector planes. This leads to improved accuracy in estimating the reference phase maps since the low-dimensional representation averages out the errors in individual observations. Our approach, in its minimal form, needs projection of a single (grid) pattern on only two reference (camera calibration) planes instead of multiple sinusoidal patterns, thereby speeding up the system calibration process. Our approach is highly flexible and does not need precise placement of reference planes. Further, we also use the projective invariance of the cross-ratio to solve for 3D point location in the scanning phase. The results presented demonstrate the accuracy of the approach for geometric calibration. In addition, we introduced a cubic spline-based approach to model the geometric error induced in the 3D reconstruction due to radiometric non-linearities of the projector. The utility of our approach to radiometric correction is effectively demonstrated on real data.

\section{References}

1. Dhillon, D.S., Govindu, V.M.: On using projective relations for calibration and estimation in a structured-light scanner. In: Bebis, G., Boyle, R., Parvin, B., Koracin, D., Juno, Y., Wang, J., Wang, J-X., Wang, J., Pajarola, R., Lindstrom, P., Hinkenjann, A., Encarnacao, M.L., Silva, C.T., Coming, D. (eds.) Advances in Visual Computing, LNCS Series, vol. 5875, pp. 831-842. Springer, Berlin, Heidelberg (2009)

2. Fofi, D., Salvi, J., Mouaddib, E.: Uncalibrated reconstruction: an adaptation to structured light vision. Pattern Recognit. 36(7), 16311644 (2003)

3. Furukawa, R., Kawasaki, H.: Dense 3D reconstruction with an uncalibrated stereo system using coded structured light. In: Proceedings of the IEEE Computer Society Conference on Computer Vision and Pattern Recognition (CVPR)-workshops (2005). doi: 10. 1109/CVPR.2005.440

4. Li, Y.F., Lu, R.S.: Uncalibrated Euclidean 3-D reconstruction using an active vision system. IEEE Trans. Robot. Autom. 20(1), 15-25 (2004)

5. Zhang, S., Huang, P.S.: Novel method for structured light system calibration. Opt. Eng. SPIE 45(8), 083601 (2006). (pp. 1-8)

6. Peng, T., Gupta, S.K.: Model and algorithms for point cloud construction using digital projection patterns. J. Comput. Inf. Sci. Eng. 7(4), 372-381 (2007)

7. Ishiyama, R., Okatani, T., Deguchi, K.: Precise 3-D measurement using uncalibrated pattern projection. In: IEEE International Con- ference on Image Processing, San Antonio, TX, USA, pp. 225-228 (2007)

8. Moreno, D., Taubin, G.: Simple, accurate, and robust projectorcamera calibration. In: IEEE International Conference on 3D Imaging, Modeling, Processing, Visualization and Transmission (3DIMPVT), Zurich, Switzerland, pp. 464-471 (2012)

9. Orghidan, R., Salvi, J., Gordan, M., Florea, C., Joan Batlle, $\mathrm{J}$.:Structured light self-calibration with vanishing points. Mach. Vis. Appl. 25(2), 489-500 (2014)

10. Martynov, I., Kamarainen, J., Lasse Lensu, L.: Projector calibration by "inverse camera calibration". In: Heyden, A., Kahl, F. (eds.) Image Analysis, LNCS Series, vol. 6688, pp. 536-544. Springer, Berlin, Heidelberg (2011)

11. Li, T., Hu, F., Geng, Z.: Geometric calibration of a camera-projector 3D imaging system. In: Proceedings of the 10th International Conference on Virtual Reality Continuum and Its Applications in Industry, pp. 187-194. ACM (2011)

12. Fernandez, S., Joaquim Salvi, J.: Planar-based camera-projector calibration. In: 2011 7th International Symposium on Image and Signal Processing and Analysis (ISPA), pp. 633-638. IEEE (2011)

13. Park, S., Go Gwang Park, G.: Active calibration of cameraprojector systems based on planar homography. In: 2010 20th International Conference on Pattern Recognition (ICPR), pp. 320-323. IEEE (2010)

14. Pan, B., Kemao, Q., Huang, L., Asundi, A.: Phase error analysis and compensation for nonsinusoidal waveforms in phase-shifting digital fringe projection profilometry. Opt. Lett. 34(4), 416-418 (2009)

15. Liu, K., Wang, Y., Lau, D., Hao, Q., Hassebrook, L.: Gamma model and its analysis for phase measuring profilometry. JOSA A 27(3), 553-562 (2010)

16. Rocchini, C., Cignoni, P., Montani, C., Pingi, P., Scopigno, R.: A low cost 3D scanner based on structured light. Comput. Graph. Forum 20(3), C-299-C-308 (2001)

17. Salvi, J., Pages, J., Batlle, J.: Pattern codification strategies in structured light systems. Pattern Recognit. 37(4), 827-849 (2004)

18. Hartley, R., Zisserman, A.: Multiple View Geometry in Computer Vision, 1st edn. Cambridge University Press, Cambridge (1999)

19. Bouguet, J.: Caltech Calibration Toolkit. http://www.vision. caltech.edu/bouguetj/calib_doc/. Accessed 23 Feb 2015

20. Zhang, Z.: A flexible new technique for camera calibration. IEEE Trans. Pattern Anal. Mach. Intell. 22(11), 1330-1334 (1998)

21. Torr, P.H.S.: Bayesian model estimation and selection for epipolar geometry and generic manifold fitting. Int. J. Comput. Vis. 50(1), 35-61 (2002)

22. Torr, P.H.S.: Structure and Motion Toolkit in MATLAB. http:// www.mathworks.com/matlabcentral/fileexchange/authors/12514. Accessed 23 Feb 2015

23. MeshLab Toolkit. http://meshlab.sourceforge.net/. Accessed 23 Feb 2015

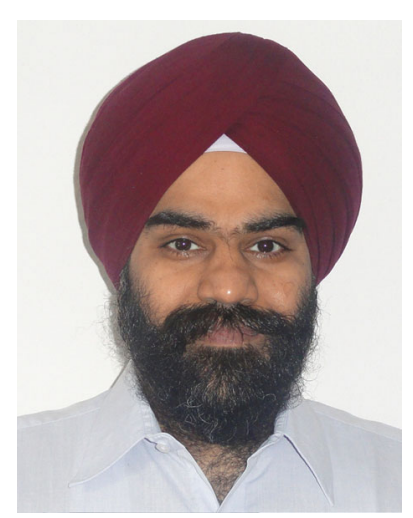

Daljit Singh Dhillon obtained a M.Sc (Engg.) degree from the Department of Electrical Engineering, Indian Institute of Science, Bengaluru, India. He is currently enrolled for a Ph.D. degree with the Computer Graphics Group at the Institute of Computer Science and Applied Mathematics, University of Bern, Bern, Switzerland. Daljit Singh's research interests include topics in computer vision and graphics. 


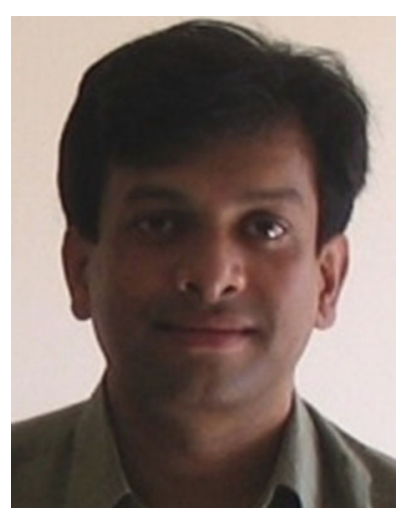

Venu Madhav Govindu obtained a Ph.D. degree in Electrical Engineering from the University of Maryland, College Park, USA. $\mathrm{He}$ is an Assistant Professor in the Department of Electrical Engineering, Indian Institute of Science, Bengaluru, India. His research interests are in geometry and statistical inference problems in computer vision. 